\title{
Numerical Simulation of Taylor Flow in the Entrance Region of Microchannels
}

\author{
Amin Etminan, Yuri S. Muzychka, Kevin Pope \\ Faculty of Engineering and Applied Science, Memorial University of Newfoundland \\ St. John's, NL A1B 3X5, Canada \\ aetminan@mun.ca; yurim@mun.ca; kpope@mun.ca
}

\begin{abstract}
Computational Fluid Dynamics (CFD) has been widely employed by investigators to simulate Taylor flow in microchannels. High-resolution images captured by numerical techniques reveal significant details of an ultra-thin liquid film around the gas bubble. The interface between the liquid and the gas phases is a decisive factor in order to determine the flow pattern, the gas bubble profile, the bubble length-separation distance, the slug length, and so forth. Since the thickness of the liquid film is on the order of 10-15 $\mu \mathrm{m}$ for low capillary number flows, the mesh generation requires careful modeling to capture it and transport phenomena, such as momentum and heat. The present study is to develop a model of a transient Taylor flow in a two-dimensional microchannel using commercial ANSYS Fluent software. The Volume of Fluid ( $\mathrm{VoF}$ ) method was employed to simulate the interface between two phases, i.e., air and water. A comprehensive grid study was carried out to identify a sufficiently fine mesh to capture the film layer around the gas bubble in the uniform film thickness region. The curvatures of the nose and the tail of the gas bubbles were studied during traveling through the channel. Variations of the lengths of slugs and plugs were also measured as the functions of time. The numerical results were validated by available correlations of liquid film thickness, and slug length in previous studies.
\end{abstract}

Keywords: Gas-Liquid; Microchannel; Numerical Simulation; Slug Length; Taylor Flow; Two-Phase Flow.

\section{Introduction}

Micro-structured mechanical systems have been emerging in more compact industrial applications. Specifically, a microchannel has been designed to minimize pressure drop and maximize transport phenomena in most energy-related microfluidics purposes, such as heat exchangers, fuel cells, and microreactors. A typical gas-liquid two-phase flow refers to a mixture of two distinct phases as long as the phases are separated by the interface lines. Such flows remain in the laminar flow regime due to predominant viscous and surface tension forces in them, which simplifies the numerical simulations without any turbulence modeling. The patterns of gas-liquid two-phase flows in microchannels are highly dependent on the transport phenomena, the type of channels, the phase superficial velocities, and the fluid properties, such as density, viscosity, and surface tension. Considering the remarkable low-velocity flow and very small-diameter channels, the gravitational and inertia forces are insignificant compared to the surface tension and the viscous forces. Various flow patterns, such as bubbly, Taylor, churn, annular, stratified, and wavy have been reported by investigators [1-8]. An informative way to describe the patterns of multiphase flows is to map the flow pattern on an $\mathrm{x}-\mathrm{y}$ graph, where the axes could be volumetric flow rate ratios, phase superficial velocities, and non-dimensional numbers such as: Reynolds (Re), capillary (Ca), and Weber (We). The slug pattern occupies a large region in these maps. Consequently, accurate predictions of the gas bubble formation, growth, and propagation in microchannels are required, and calculations of some predominant flow parameters including void fraction, pressure drop, and liquid film thickness are taken into account.

The interaction structures of different phases and between phases and the solid walls not only establish the patterns of multiphase flows but also predict the amount of pressure drop through the microchannel. Having an optimized microchannel with the lowest pressure drop is required to predict the value of the frictional pressure drop at first. Reference [9] considered the total pressure drop as a summation of a single-phase flow pressure drop component and an interaction pressure drop component. Some scholars decomposed the pressure drop in a unit cell into three components, namely, pressure drop in a liquid slug, pressure drop in the flat film region, and pressure drop at the nose and tail transitional regions of the gas bubble $[5,10-11]$. 
In Taylor flows, gas bubbles always are surrounded by a thin layer of continuous flow. Even though the thickness of the liquid is thin, it significantly affects the pressure drop, the transport phenomena, and the curvatures of the bubble. The bubble, with curvatures at both ends follows the semi hemispherical profiles. This type of curvature is well demonstrated in both experimental and analytical data as well [12-16]. A transition region that does not obey a semi hemispherical meniscus links the nose or tail cap with a flat/steady length of liquid film thickness. The measurement of liquid thickness in the flat region has recently appeared in major studies concerning gas-liquid flows in microchannels, which has been correlated as a function of non-dimensional numbers $\mathrm{Ca}$, Re, and We. Some correlations for film thickness are compiled in Table 1, which will be addressed later to validate the results of the present study.

Table 1: The available correlations for the liquid film thickness in Taylor flow.

\begin{tabular}{lll}
\hline Ref. & Correlation for $(\delta / \mathrm{R})$ & Film Thickness $(\mu \mathrm{m})$ \\
\hline Fairbrother and Stubbs (1935) & $0.5 \mathrm{Ca}^{1 / 2}$ & 9.82 \\
Bretherton (1961) & $1.338 \mathrm{Ca}^{2 / 3}$ & 11.26 \\
Schwartz et al. (1986) & $1.338 \mathrm{Ca}^{2 / 3}$ & 11.26 \\
Ratulowski and Chang (1989) & $1.338 \mathrm{Ca}^{2 / 3}$ & 11.26 \\
Aussillous and Quéré (2000) & $1.34 \mathrm{Ca}^{2 / 3} /\left(1+3.35 \mathrm{Ca}^{2 / 3}\right)$ & 10.14 \\
Kreutzer et al. (2001) & $0.36\left(1-\mathrm{e}^{\left.-2.13 \mathrm{Ca}^{0.52}\right)}\right.$ & 12.63 \\
Han and Shikazono (2009) & $1.34 \mathrm{Ca}^{2 / 3} /\left(1+3.13 \mathrm{Ca}^{2 / 3}+0.504 \mathrm{Ca}^{0.672} \mathrm{Re}^{0.589}-0.352 \mathrm{We}^{0.629}\right)$ & 10.60 \\
Klaseboer et al. (2014) & $1.338 \mathrm{Ca}^{2 / 3} /\left(1+3.732 \mathrm{Ca}^{2 / 3}\right)$ & 10.00 \\
Ni et al. (2017) & $1.34 \mathrm{Ca}^{2 / 3} /\left(1+3.13 \mathrm{Ca}^{2 / 3}+0.504 \mathrm{Ca}^{0.672} \mathrm{Re}^{0.589}-0.305 \mathrm{We}^{0.664}\right)$ & 10.05 \\
\hline
\end{tabular}

This paper focuses on a numerical study of air-water flow in a two-dimensional microchannel. The gas bubble formation at a concentric junction is investigated to show how a gas bubble generates, propagates, and moves through the microchannel. The gas bubble profile, the head and nose curvatures, the liquid film thickness, the lengths of liquid slugs, gas bubbles, and steady or flat film thickness are calculated utilizing a fine enough mesh throughout the computational domain to capture interface and transport phenomena. A schematic of a two-dimensional microchannel is illustrated in Figure 1. As is shown, the airflow enters at the core of the inlet cross-section and the water flow enters at an annular cross-section around the core. Air flows in over $70 \%$ of the inlet diameter which means the same volumetric flow rates for both flows in an axisymmetric microchannel. Then, a mixture of phases leaves the channel at the outlet.

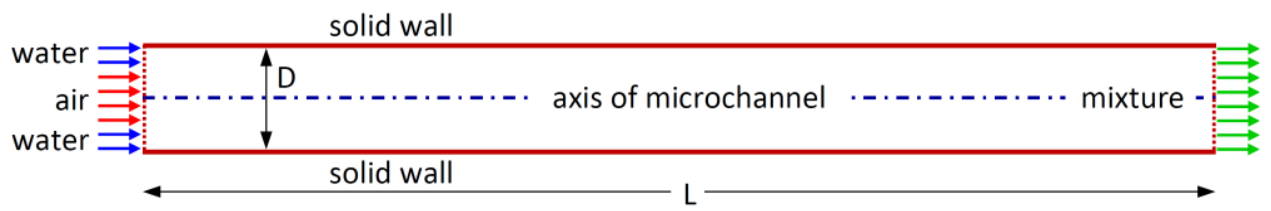

Fig. 1: Schematic of two-dimensional microchannel used in the simulations.

\section{Governing Equations and Mathematical Model}

An incompressible gas-liquid two-phase flow is assumed in a two-dimensional microchannel where each phase is a Newtonian fluid with constant thermophysical properties. The gas and liquid phases are immiscible and phase change does not occur within the microchannel. Therefore, the continuity and momentum equations take on the following forms:

$$
\begin{gathered}
\nabla \cdot \vec{u}=0 \\
\frac{\partial(\rho \vec{u})}{\partial t}+\nabla \cdot(\rho \vec{u} \vec{u})=-\nabla p+\nabla \cdot\left[\mu\left(\nabla \vec{u}+(\nabla \vec{u})^{T}\right)\right]+\rho \vec{g}+\overrightarrow{F_{S}}
\end{gathered}
$$


where $g, t, u, p, \mu$ and $\rho$ denote the gravity acceleration, time, velocity, pressure, the dynamic viscosity and the density, respectively. The last term in the momentum equation represents the surface tension force per unit area, which is approximately considered as a body force surrounding the interface line between the phases [18]:

$$
\overrightarrow{F_{S}}=\sigma \kappa \delta \vec{n}
$$

where $\sigma, \kappa, \delta$, and $\vec{n}$ represent the surface tension force, the radius of the curvature or surface normal (viewed from the gas bubble), the Dirac delta function, and the unit normal vector on the interface line, respectively.

An explicit approach is chosen to discretize the time steps by a standard finite-difference interpolation scheme applied to the volume fractions at the previous time step. In particular, ANSYS Fluent can compute the values of the face fluxes near the interface line by interpolation either using an interface reconstruction or a Finite Volume (FV) discretization scheme. The reconstruction scheme obtains the amount of flux on the faces whenever a cell is filled with a phase. The finite volume discretization approaches can be only employed with an explicit VoF method using first-order upwind or second-order upwind, and QUICK algorithms. The VoF method calculates a time step based on the transient time characteristic over a control volume, which is not necessarily equal in other governing equations. In the vicinity of the interface region, the ratio of the volume of each cell and the sum of the outgoing flux from the faces of the finite volume leads to the time taken to empty a cell. The Courant number (Co) is defined as $\Delta \mathrm{t} U / \Delta \mathrm{x}$ where the $\Delta \mathrm{x}$ and $\mathrm{U}$ represent the grid size and fluid velocity, respectively. In the following simulations, the maximum value of the Co is 0.25 , and a fixed time step of $10^{-6}$ is employed to reduce total computational time unless it causes an unexpected increase in global Courant number. To prevent the divergence of the solution in such cases, a variable time step between $10^{-6}$ and $10^{-7}$ is beneficial, particularly at the moments of gas bubble breaking off.

On the other hand, the implicit approach does not have any limitation on the Courant number making it suitable for a larger grid size-poor quality mesh- and a longer time step compared to the explicit approach. But, its higher numerical diffusion in the interface region predicts the interface curvature between phases rough and inaccurate which are illustrated in Figure 2. As is seen, the explicit approach allows the solver to employ a Geo-Reconstruct as the volume fraction discretization scheme resulting in a clear, and crisp interface curvature with no numerical diffusion. For example, the Modified HRIC scheme employed by the implicit approach, conversely, shows much more numerical diffusion and is not able to predict an accurate interface curvature at all. The Modified HRIC creates a thicker interface, a longer air bubble, and remarkable numerical diffusion on the axis inside the bubble. This weakness causes divergence of the solution at the moment of the air bubble breaking off. Consequently, the explicit approach along with the Geo-Reconstruct discretization scheme equip the following simulations.

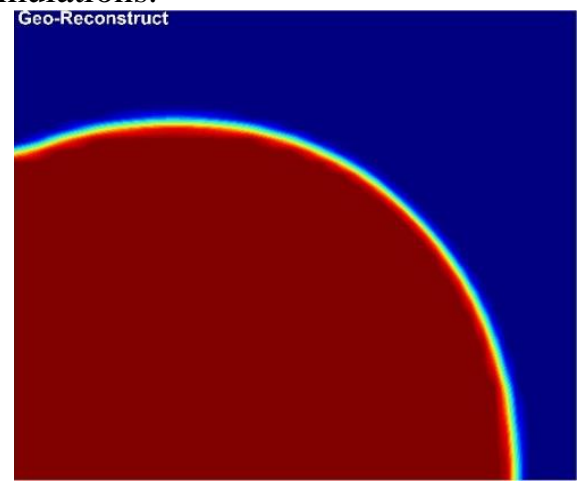

(a)

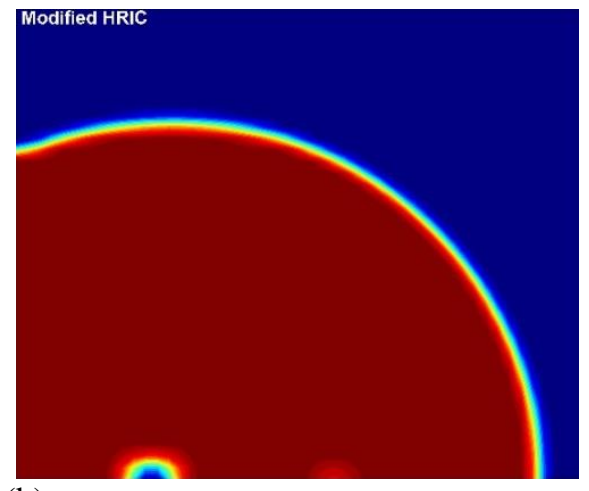

(b)

Fig. 2: Prediction of the interface line by (a) explicit, and (b) implicit approaches. (The air is colored carmine red and the water is colored navy blue)

\section{Flow and Boundary Conditions}

This study is carried out to perform a systematic numerical simulation of two-phase flow in a two-dimensional microchannel. The geometric parameters of the microchannel are depicted in Figure 1 where the channel diameter (D) is 0.5 $\mathrm{mm}$ and the channel length (L) is $5 \mathrm{~mm}$. Based on fluid mechanics textbooks, the entrance length of channels is 10 times the 
diameter [18]. Under this circumstance, the considered two-phase flow never approaches a fully developed flow, and the velocity inlet boundary condition is assumed for both phase flows at the inlet. Outlet flow boundary condition is applied at the exit plane of the channel where the gas bubbles and the liquid slugs exit simultaneously. The axisymmetric geometry of microchannel encourages the simulations to be conducted for only half of the actual computational domain resulting in lower computational time. Therefore, the axis boundary condition is set on the axis of the channel where the normal gradients for all variables are zero. Finally, the typical no-slip boundary condition is presumed at the solid wall of the microchannel. The thermophysical properties of operating fluid flows at room temperature of $25^{\circ} \mathrm{C}$ are listed in Table 2. The superficial velocities of the air and the water are $0.245 \mathrm{~m} . \mathrm{s}^{-1}$ and $0.255 \mathrm{~m} . \mathrm{s}^{-1}$, respectively. These flow conditions result in $\mathrm{Ca}, \mathrm{Re}$, and $\mathrm{We}$ values of $0.00618,279.93$, and 1.73 , respectively. The simulations are initialized to incoming air and water phases uniformly.

Table 2: Thermophysical properties of air and water used in the simulations.

\begin{tabular}{llll}
\hline Fluid & Density $\left(\mathrm{kg} \cdot \mathrm{m}^{-3}\right)$ & Dynamic Viscosity $\left(\mathrm{kg} \cdot \mathrm{m}^{-1} \cdot \mathrm{s}^{-1}\right)$ & Surface Tension $\left(\mathrm{N} \cdot \mathrm{m}^{-1}\right)$ \\
\hline Air & 1.1845 & $1.849 \times 10^{-5}$ & 0.072 \\
Water & 997.1 & $8.905 \times 10^{-4}$ & \\
\hline
\end{tabular}

\section{Discretization Procedure}

The governing equations must be discretized by the specific numerical discretization algorithms regarding the boundary conditions mentioned earlier. To avoid a de-coupling of velocity and pressure variables for Scale-Resolving Simulations (SRS), a proper algorithm must be chosen considering a few key factors: geometry of the problem, properties of fluids involved, flow regime, and activated additional models (if any). In the present study, a first order Non-Iterative Time (NIT) marching scheme is employed to reduce the amount of computational time for each time-step. The NIT does not require the outer iterations resulting in a significant reduction of computational efforts and speed up the simulations consequently [19-23]. This scheme is also beneficial of user-defined number of sub-iteration for each individual governing equation along with the correction tolerance which are set to the order of $10^{-7}$.

Least Squares cell-based, Green-Gauss cell-based, and Green-Gauss node-based approaches are available to calculate not only the gradient interpolation of the flow parameters but also secondary diffusion terms and the derivatives of velocity at the centre of cell faces. The most proper method, which is resulted in the highest accuracy, and the cheapest computation is highly problem-dependent [19, 24-25]. Eventually, Body Force Weighted, Second-Order Upwind, and Geo-Reconstruct schemes are assumed for pressure, momentum, and volume fraction interpolations, respectively to achieve the highest accuracy with minimal computational effort. Another scheme for momentum interpolation is QUICK which stands for Quadratic Upstream Interpolation for Convective Kinematics. The QUICK is using a second-order central difference for diffusion terms and a third-order central difference for convection terms. This scheme is of benefit to a weighted average of second-order upwind to interpolate scalar variables. The QUICK shows more accuracy compared to second-order upwind for computing variables on structured meshes [25-27]. Hence, the QUICK discretization scheme has been employed in the following simulations. In addition to an appropriate time step and the number of iterations per each time step, a careful under relaxation factors adjustment is required to make a robust solver and prevent a divergence solution. A poor-quality mesh can make numerical instabilities during solutions.

\section{Results and Discussion}

The first step of each computational fluid dynamics problem is generating proper mesh to launch simulations. A proper grid resolution is significantly important in numerical studies to capture transport phenomena, particularly Taylor flow in microchannels. In the lack of geometric complexities of the present study, a quadratic mesh is utilized to discretize the two-dimensional microchannel. Awareness of approximate film thickness enables mesh generation to be more precise. Therefore, the amount of stable non-dimensional film thickness under present circumstances is computed by different empirical correlations, which are earlier compiled in Table 1. As is seen, the film thickness ranges from 9 to 13 microns and will be used later to establish an accurate fine grid. Most of the proposed correlations are based on experimental data of air-water two-phase flow except [28]. It should be notified that the thicknesses of the liquid film in 
Table 1 are in a fully developed Taylor flow where the measuring location is far enough from the junction and the film thickness remains constant. While the present study is analyzing a developing Taylor flow in the entrance region of the microchannel.

Three discretization approaches can be considered, namely, coarse, fine-coarse, and fine. The coarse approach divides the whole domain into square-shaped cells starting with $25 \times 25 \mu \mathrm{m}$ cells as the first trial. Figure 3 represents the liquid volume fraction for a coarse-size mesh of 25 microns (case I) at every millisecond to show how the air-bubbles emerge and move downstream. Since the size of cells is greater than approximate film thickness, the boundary layer, liquid film, and interface near the wall cannot be properly captured. As is expected, the boundary layer is not realized by the simulations and the dryout is observed at the wall. It means that the liquid film is no present around the air bubble and the air is in direct contact with the channel's wall. Furthermore, the air-water interface becomes thick, sharp, and not semi hemispherical curvature at the rear end.

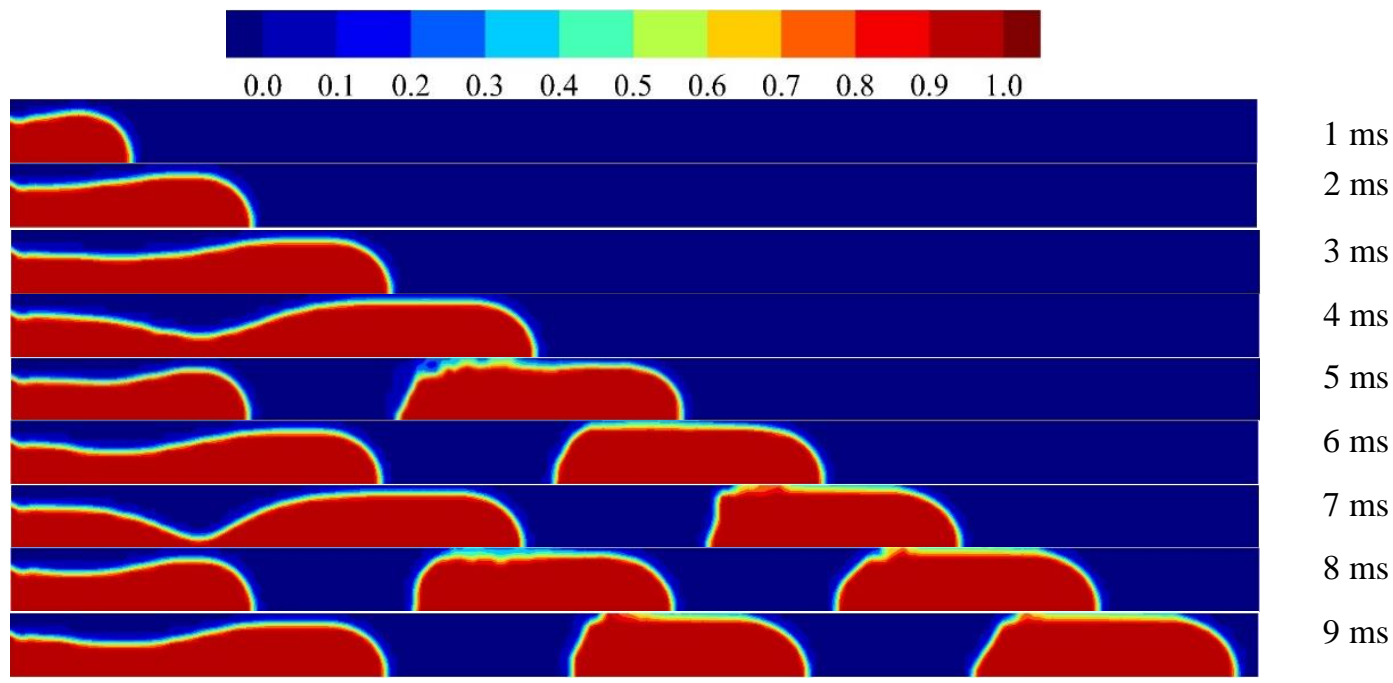

Fig. 3: Liquid volume fraction plots for a uniform coarse-sized mesh of $25 \mu \mathrm{m}$.

(The air is colored carmine red and the water is colored navy blue)

Therefore, grid refinement is required to enable the numerical simulations to sense the solid wall, shape the interface line, and form the bullet-shaped bubbles successfully. Four other refinements are assumed to realize an independent fine mesh which are tabulated in Table 3 at $9 \mathrm{~ms}$. As is mentioned earlier, case I is not able to capture film thickness and only two bubbles are generated over $9 \mathrm{~ms}$. By halving the grid size, case II, three shorter air bubbles are observed, but the liquid film thickness is not still captured. Therefore, neither of the cases I and II are able to capture film thickness nor interface line properly. Case III captures liquid film thickness successfully and three bullet shape air bubbles have been generated over 9 ms when the lengths of bubbles are decreased with time marching. Further decrease in the grid size, establish an approximate same flow pattern. The grid refinement from case III to IV makes a 3.7\% deviation, while from case IV to V leads to a $1.4 \%$ variation. It seems that the flow patterns, the lengths and the numbers of slugs and plugs are not dependent on the mesh refinement further than case IV. Therefore, the following numerical discussion, such as refinement near the wall have been carried out on case IV.

Table 3: Quantitative aspects of the hydrodynamics of flow to find an independent coarse grid.

\begin{tabular}{|c|c|c|c|c|c|c|c|c|c|c|}
\hline \multirow[t]{2}{*}{ Case } & \multirow{2}{*}{$\begin{array}{l}\text { Mesh Size } \\
(\mu \mathrm{m})\end{array}$} & \multicolumn{3}{|c|}{ Bubble Breaking off (ms) } & \multirow{2}{*}{$\begin{array}{l}\text { Film Thickness } \\
(\mu \mathrm{m})\end{array}$} & \multicolumn{2}{|c|}{ Slug Length $\left(\mathrm{L}_{\mathrm{s}} / \mathrm{D}\right)$} & \multicolumn{3}{|c|}{ Bubble Length $\left(\mathrm{L}_{\mathrm{b}} / \mathrm{D}\right)$} \\
\hline & & $3^{\text {rd }}$ & $2^{\text {nd }}$ & $1^{\text {st }}$ & & $2^{\text {nd }}$ & $1^{\mathrm{st}}$ & $3^{\text {rd }}$ & $2^{\text {nd }}$ & $1^{\mathrm{st}}$ \\
\hline $\mathrm{I}$ & 25 & ----- & 7.04 & 4.23 & fully dry-out & ----- & 1.32 & ----- & 2.11 & 1.89 \\
\hline II & 12.5 & 8.84 & 6.08 & 3.74 & partially dry-out & 1.22 & 1.16 & 1.72 & 1.74 & 1.91 \\
\hline III & 8.33 & 8.78 & 6.29 & 3.62 & 10.41 & 1.14 & 1.12 & 1.70 & 1.72 & 1.84 \\
\hline IV & 6.25 & 8.45 & 6.10 & 3.57 & 10.24 & 1.12 & 1.09 & 1.68 & 1.71 & 1.82 \\
\hline $\mathrm{V}$ & 5 & 8.43 & 6.08 & 3.52 & 10.23 & 1.12 & 1.10 & 1.67 & 1.71 & 1.81 \\
\hline
\end{tabular}


In the fine-coarse approach, the presence of the solid walls encourages the mesh generation process to employ a non-uniform distribution perpendicular to the flow direction. Therefore, the generated mesh should be refined near the walls to be able of capturing boundary layer and liquid film thickness adequately. Then, a uniform mesh distribution is considered along the axis of the microchannel. This grid generation is beneficial to reduce the dispersion of numerical calculations in the region of the gas-liquid interface. Over a thickness of $15 \mu \mathrm{m}$ near the walls, 5, 6, and 7 cells are considered to enable the simulations of capturing film thickness successfully. The lengths of the cells set to a constant value of $6.25 \mu \mathrm{m}$. It is expecting these fine grid sizes near the wall and the coarse grid in the core region of the microchannel not only helps to capture the film thickness successfully but also reduces the computational time significantly. The corresponding total numbers of cells are 34400, 35200, and 36000. With these sizes of the meshes, a linear slope of the interface line between two phases at the inlet can be assumed to calculate the advection of each phase at the cell faces [29]. The refinements have resulted in the average film thicknesses of $11.67,10.94$, and $10.85 \mu \mathrm{m}$ for mesh sizes of 3, 2.5, and $2.43 \mu \mathrm{m}$, respectively (see Table 4). Since an insignificant change of $0.8 \%$ is observed by mesh refining from 2.5 to $2.43 \mu \mathrm{m}$, the grid size of $2.5 \mu \mathrm{m}$ furnishes the numerical results with reasonable accuracy. An ultrarefined mesh not only enhances the computational time but also causes non-physical pressure jump in the film region as observed by [24]. Eventually, the following discussion has been conducted for a fine-coarse grid case IV over the core region and fine case II over a thickness of $15 \mu \mathrm{m}$ near the wall. This fine-coarse grid which is aligned with the flow minimizes the false diffusion and truncation error [26-27].

Table 4: Quantitative aspects of the hydrodynamics of flow to find an independent fine grid near the wall.

\begin{tabular}{llllllllll}
\hline Case & Mesh Size $(\mu \mathrm{m})$ & \multicolumn{3}{c}{ Film Thickness $(\mu \mathrm{m})$} & \multicolumn{3}{c}{ Slug Length $\left(\mathrm{L}_{\mathrm{s}} / \mathrm{D}\right)$} & \multicolumn{3}{c}{ Bubble Length $\left(\mathrm{L}_{\mathrm{b}} / \mathrm{D}\right)$} \\
\cline { 3 - 9 } & & $3^{\text {rd }}$ & $2^{\text {nd }}$ & $1^{\text {st }}$ & $2^{\text {nd }}$ & $1^{\text {st }}$ & $3^{\text {rd }}$ & $1^{\text {st }}$ \\
\hline I & 3 & 12.50 & 11.50 & 11.00 & 1.15 & 1.14 & 1.79 & 1.76 & 1.85 \\
II & 2.5 & 10.75 & 11.14 & 10.94 & 1.16 & 1.14 & 1.78 & 1.75 & 1.85 \\
III & 2.43 & 10.62 & 11.10 & 10.83 & 1.16 & 1.13 & 1.78 & 1.75 & 1.86 \\
\hline
\end{tabular}

Figure 4 shows a time history of volume fraction for a non-uniform fine-sized mesh, case II, at every one millisecond. As is seen a train of air bubbles which colored by carmine red follows an evolution process involving hydrodynamics phenomena, such as emersion, elongation, filling up, necking, breaking off, and moving. The bullet-like bubbles travel downstream with an insignificant change in the nose curvature, while the rear curvature experiences an undulation over one millisecond after break-off moment. This pattern was also perceived by [24]. According to Table 4, maximum variations of $5 \%, 1.7 \%$, and $3.6 \%$ are observed for bubble length, slug length, and film thickness, respectively. It is evident from the results that the bubble formation process occurs frequently with a similar length and shape.

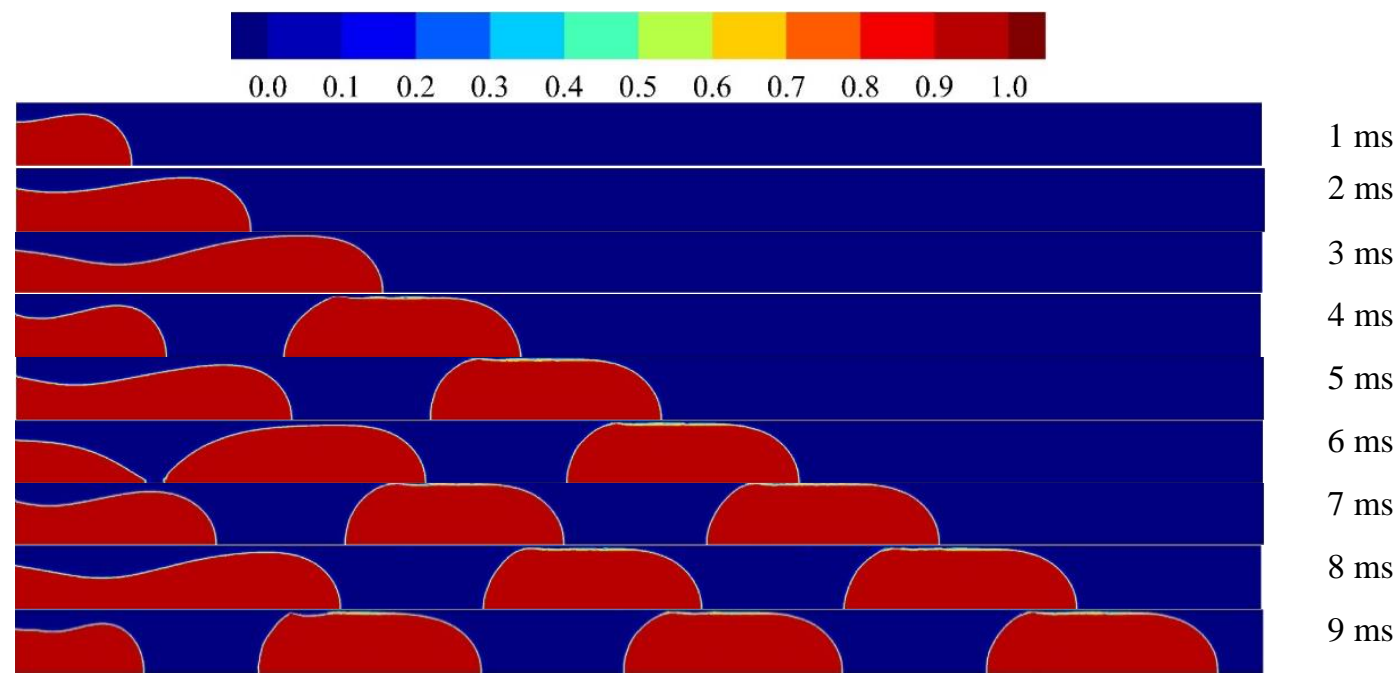

Fig. 4: Liquid volume fraction plots for a non-uniform fine-sized, case II.

(The air is colored carmine red and the water is colored navy blue) 
The developing nature of assumed Taylor flow causes some ripples at the liquid film thickness which are shown by the enlarged views of the film thickness regions of three air bubbles in Figure 5. It is worth pointing out that the ripples disappearing as the bubble moves downstream-time marching.

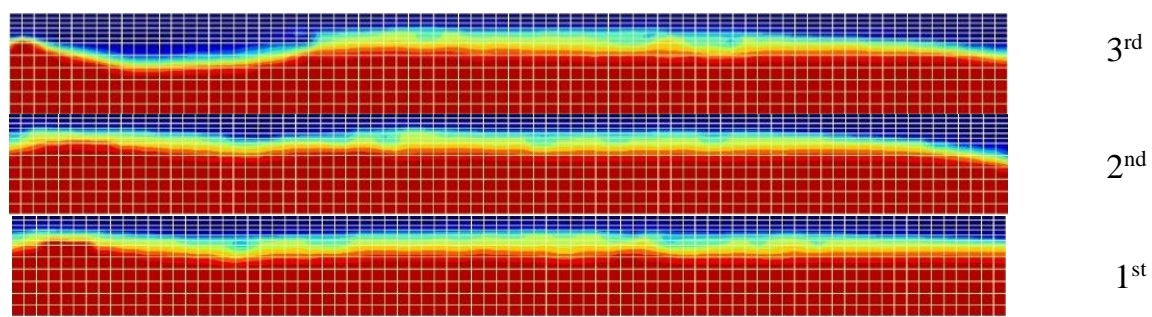

Fig. 5: Zoomed-in views of liquid film thickness regions of three air bubbles at $9 \mathrm{~ms}$.

(The air is colored carmine red and the water is colored navy blue)

Likewise, the length of flat film thickness region becomes longer-the nose and tail transition lengths become shorteras the bubble travels to the outlet which tabulated in Table 5. The variations of the lengths of transition and flat regions become smaller as the bubble moving. The semi hemispherical meniscus at the nose and rear parts of the bubbles can be approximately fitted by two spheres at the nose and tail with a radius of R1 and R2, respectively. As is also seen in Table 5, the radius of the nose curvature is less than that of the tail but the bullet-like profile of the second bubble remained almost constant.

Table 5: Comparison of the air bubble shapes and the geometric details.

\begin{tabular}{llllll}
\hline \multirow{2}{*}{ Bubble } & \multicolumn{2}{l}{ Meniscus Diameter $(\mu \mathrm{m})$} & \multicolumn{2}{l}{ Transition Length $(\mu \mathrm{m})$} & Flat Region Length \\
\cline { 2 - 5 } & $\mathrm{R} 1$ & $\mathrm{R} 2$ & nose & tail & $(\mu \mathrm{m})$ \\
\hline $1^{\text {st }}$ & 210 & 240 & 120 & 109 & 502 \\
$2^{\text {nd }}$ & 210 & 240 & 166 & 158 & 399 \\
$3^{\text {rd }}$ & 200 & 240 & 227 & 275 & 265 \\
\hline
\end{tabular}

\section{Conclusions}

This study performed a series of numerical simulations of an air-water Taylor flow under developing circumstances in a microchannel with a circular cross-sectional area. A comprehensive grid study was conducted by three grid approaches, such as coarse, fine-coarse, and fine were examined to discover a mesh that makes the results independent of grid size. A systematic grid generation study was also investigated to obtain an optimized mesh providing accurate results. It was argued that the mesh size caused different interactions between two phases and the channel wall, i.e., dry-out, partially dry-out, and fully wetted to explain whether the wall was kept dry or wet. Investigation of the grid reveals a significant role of the number of grids over the liquid film thickness region leading to a smooth and crisp interface. The results also showed that the liquid film thickness of the bubbles remained almost constant, but the length of the flat film region increased as the air bubble moving downstream. It is suggesting that the viscosity effects of different dispersed flows on the flow pattern and hydrodynamics facts can be pursued in the future works, particularly in a more complex microchannel path.

\section{Acknowledgements}

The authors appreciate the financial support from the Natural Sciences and Engineering Research Council of Canada (NSERC) grant NSERC DG 200439.

\section{References}

[1] Y. Taitel, D. Bornea and A.E. Dukler, "Modelling flow pattern transitions for steady upward gas-liquid flow in vertical tubes," AlChE Journal, vol. 26, no. 3, pp. 345-354, 1980.

[2] A. Kawahara, P.M.-Y. Chu ng and M. Kawaji, "Investigation of two-phase flow pattern, void fraction and pressure drop in a microchannel," International Journal of Multiphase Flow, vol. 28, pp. 1411-1435, 2002.

[3] M.K. Akbar, D.A. Plummer and S.M. Ghiaasiaan, "On gas-liquid two-phase flow regimes in microchannels," International Journal of Multiphase Flow, vol. 29, no. 5, pp. 855-865, 2003. 
[4] T. Cubaud and C.M. Ho, "Transport of bubbles in square microchannels," Physics of Fluids, vol. 16, no. 12, pp. 45754585, 2004.

[5] M.T. Kreutzer, F. Kapteijn, J.A. Moulijn, C.R. Kleijn and J.J. Heiszwolf, "Inertial and interfacial effects on pressure drop of Taylor flow in capillaries," American Institute of Chemical Engineers, vol. 51, no. 9, pp. 2428-2440, 2005.

[6] M.N. Kashid, A. Renken and L. Kiwi-Minsker, "Influence of flow regime on mass transfer in different types of microchannels," Industrial \& Engineering Chemistry Research, vol. 50, no. 11, pp. 6906-6914, 2011.

[7] J.M. Bolivar and B. Nidetzky, "Multiphase biotransformations in microstructured reactors: opportunities for biocatalytic process intensification and smart flow processing," Green Processing and Synthesis, vol. 2, no. 6, pp. 541-559, 2013.

[8] A.A. Yagodnitsyna, A.V. Kovalev and A.V. Bilsky, "Flow patterns of immiscible liquid-liquid flow in a rectangular microchannel with T-junction," Chemical Engineering Journal, vol. 303, pp. 547-554, 2016.

[9] E. Walsh, Y. Muzychka, P. Walsh, V. Egan and J. Punch, "Pressure drop in two phase slug/bubble flows in mini scale capillaries," International Journal of Multiphase Flow, vol. 35, no. 10, pp. 879-884, 2009.

[10] T.S. Fouilland, D.F. Fletcher and B.S. Haynes, "Film and slug behaviour in intermittent slug-annular microchannel flows," Chemical Engineering Science, vol. 65, no. 19, pp. 5344-355, 2010.

[11] Y. Song, F. Xin, G. Guangyong, S. Lou, C. Cao and J. Wang, "Uniform generation of water slugs in air flowing through superhydrophobic microchannels with T-junction," Chemical Engineering Science, vol. 199, pp. 439-450, 2019.

[12] F.P. Bretherton, "The motion of long bubbles in tubes," Journal of Fluid Mechanics, vol. 10, no. 2, pp. 166-188, 1961.

[13] G.I. Taylor, "Deposition of a viscous fluid on the wall of a tube," Journal of Fluid Mechanics, vol. 10, no. 2, pp. 161$165,1961$.

[14] P. Aussillous and D. Quéré, "Quick deposition of a fluid on the wall of a Tube," Physics of Fluids, vol. 12, no. 10, pp. 2367-2371, 2000.

[15] H. Fujioka and J.B. Grotberg, "The steady propagation of a surfactant-laden liquid plug in a two dimensional channel," Physics of Fluids. vol. 17, pp. 082102, 2005.

[16] Y. Han and N. Shikazono, "Measurement of the liquid film thickness in micro tube slug flow," International Journal of Heat and Fluid Flow, vol. 30, no. 5, pp. 842-853, 2009.

[17] J.U. Brackbill, D.B. Kothe and C. Zemach, "A continuum method for modeling surface tension," Journal of Computational Physics, vol. 100, no. 2, pp. 335-354, 1992.

[18] F.M. White, Fluid Mechanics. McGraw-Hill, 2011.

[19] ANSYS Fluent 18.1.0 Users' Guide, SAS Inc, 2017.

[20] S. Armsfield and R. Street, "The Fractional-Step Method for the Navier-Stokes Equations on Staggered Grids: Accuracy of Three Variations," Journal of Computational Physics, vol. 153, no. 2, pp. 660-665, 1999.

[21] J.B. Perot, "An Analysis of the Fractional Step Method," Journal of Computational Physics, vol. 108, no. 1, pp. 51-58, 1993.

[22] J.K. Dukowicz and A.S. Dvinsky, "Approximate Factorization as a High-Order Splitting for the Implicit Incompressible Flow Equations," Journal of Computational Physics, vol. 102, no. 2, pp. 336-347, 1992.

[23] J.B. Bell, P. Colella and H.M. Glaz, "Second-Order Projection Method for the Incompressible Navier-Stokes Equations," Journal of Computational Physics, vol. 85, no. 2, pp. 257-283, 1989.

[24] R. Gupta, D.F. Fletcher, B.S. Haynes, “On the CFD modelling of Taylor flow in microchannels," Chemical Engineering Science, vol. 64, no. 12, pp. 2941-2950, 2009.

[25] D.G. Holmes and S.D. Connell, "Solution of the 2D Navier-Stokes Equations on Unstructured Adaptive Grids," in 9th Computational Fluid Dynamics Conference, Buffalo, NY, USA, 1989, pp. 25.

[26] S.V. Patankar, Numerical Heat Transfer and Fluid Flow. Taylor \& Francis, 1980.

[27] H.K. Versteeg and W. Malalasekera, An Introduction to Computational Fluid Dynamics: the finite volume method, Harlow, Eng.; Toronto: Pearson/Prentice Hall, 2007.

[28] M.T. Kreutzer, P. Du, J.J. Heiszwolf, F. Kapteijn and J.A. Moulijn, "Mass transfer characteristics of three-phase monolith reactors," Chemical Engineering Science, vol. 56, no. 21-22, pp. 6015-6023, 2001.

[29] D.L. Youngs, "Time-dependent multi-material flow with large fluid distortion," in Numerical Methods for Fluid Dynamics, Edited by, K.W. Morton and M.J. Baines, Eds., Academic Press, New York, NY, USA, 1982, pp. $273-285$. 